\title{
Research on the Flat Design of Mobile Terminal
}

\author{
HuiHuang ${ }^{1, a}$, Junjian Liu ${ }^{2, b}$ Jianchun Shao ${ }^{3, c}$ \\ ${ }^{1}$ School of Art and Design, Jingdezhen Ceramic Institute, Jingdezhen, 333004, China \\ ${ }^{2}$ Jingdezhen Ceramic Vocational Technical College, Jingdezhen, 333400, China \\ ${ }^{3}$ Jingdezhen Ceramic Vocational Technical College, Jingdezhen, 333400, China \\ a114249216@qq.com,,48858523@qq.com, cjdzsjc@163.com
}

Key words: mobile terminal; flat; flat design

\begin{abstract}
Flattening refers to the visual effects which can cause illusion and stimulation after abandoning those gradients, shadows, highlights and so on, so as to create the graphics and shapes which look flat and simple. Through analyzing the inevitability of the return of flattening, such as the psychological impact, visual demand, the promotion of interactive design development and the intuitive and lively characteristic of the advantage of flat design, this paper proposes the design methods of flat icon of mobile terminal, which include single-line icon design, silhouette icon design, imitation seal icon design, long shadow icon design, projection icon design and gradient icon design.
\end{abstract}

\section{The Flat Design}

As a matter of fact, flattening has had a long time. Some originally designed graphics are the effect of flattening, which is restricted by the technology, aesthetic and other factors at that time. Such graphic is called the flattening because of its style and characteristics. It refers to the visual effects which can cause illusion and stimulation after abandoning those gradients, shadows highlights and so on, so as to create the graphics and shapes which look flat and simple. In fact, the flat design lays emphasis on the minimalism, advocating the aesthetics that less is more.

It can be said that flat is one of the most popular design forms now. Many mobile terminals have changed their original logo images with the effects of projection, highlight and three-dimension into flat. For example, the network logo of Microsoft's MSN has changed from the previous four-color butterfly with transparent and superimposed effect to the graphic of pure blue butterfly; the search engine giant Google has removed its previous embossing effect, and its subordinate Youtube, the world's largest video site, has also eliminated the three-dimensional transparent effect in its official Facebook and Twitter page; Greek Alpha TV has removed highlights and embossing effect, enabling the new mark of flat style.

Currently, we can see the latest news that some foreign enterprises or groups update their logos through the Internet, among which it can be found that flat logo has occupied the mainstream status and received more comments and attention. However, the trend of returning has not been so striking in domestic as in foreign countries. No matter how this trend is spreading, it is believed that some large corporate images in domestic will change with time.

\section{The Inevitability of the Return of Flattening}

The influence on psychological level. Mental activity has a great influence on the transmission of information that people have received. While constructing the complete visual feelings of people, 
different visual phenomena of people are accompanied by corresponding psychological feelings. People will actively strive to get rid of the boredom caused by some constant things, and choice of change is the most important psychological characteristic of human. To this end, the mobile terminal icon should be regularly adjusted to cater to the target's psychology with the advance of the times, thus avoiding people to feel bored psychologically. Therefore, today the icons of many companies tend to be flat according to the actual needs of various promotional carriers.

Demand on visual level. The original design of the logo is "flat", which is used to convey information as a symbol. With the progress of time and change of aesthetic ideas, the visual freshness of people on flat effect has no longer existed, so designers have added some light elements in the logo design creatively, created the stereo effect in plane space and reflected the three-dimensional effect through the light, shadow, gradient and other elements. To some extent, this is the inheritance and breakthrough of the previous design, which makes the design more in line with the aesthetic ideas. With years of development, people have begun to have aesthetic fatigue about this design, so it is necessary to find a better sense of form to replace the previous one. However, it is very difficult to make more unconventional breakthrough, so the word "return" used here can better explain its essence: to display the original thing again. In the world full of mobile terminal icons of various effects, it reflects the different feelings.

The promotion of interactive design development. With the rapid development of society and the advance of science and technology, the position of interactive design has become more and more important. Only with the interaction can the communication with the audiences be more effective, and the relevant information be transmitted. With the increasing development of interactive design, on the one hand, the color and graphic in flat logo are more suitable for screen of any size, especially for the small one, while the recognition of logos with specific effects will decrease significantly after reduction; on the other hand, flat logo design not only takes into account the principle of minimalism but also can deal with more complexities. By removing the three-dimensional effect, these designs integrate sharpness with clarity, which can enhance the operating speed of the site and make it faster and more practical. Therefore, many enterprises or groups are moving the mobile terminal icons toward the flat effect in succession based on the above reasons.

\section{The advantages of flat design - intuitive and lively}

The flattening does not simply adopt the method of flattening the picture elements or eliminating the decorative effect. No matter what style of the design is, it is to transform the traditional representative thinking into conceptual thinking, transmit it through the visual media and form a set of effective visual language system. Through the layout design of a simple picture, flat style enables the picture to express more contents. By a large number of coordinated, retro or even sharply contrastive colors, symbols and texts, it makes introductions and guidance, brings the audiences a more purely visual perception and better directs the audiences' attention to the position where designers hope they note (namely, the position of important information ), so that the audiences communicate with the frame information. For example, we can use the effect of brown paper to decorate the picture and also use gold ornaments to enhance the classical feeling of the image, thus striking a chord of the audiences on the humanistic feelings. But all this is just decorative and the conveying effect is far less than the simple and direct display of information to audiences through symbols and colors. Flat design rejects all unnecessary elements, and advocates that the audiences should communicate directly with their desired information. 


\section{The flat icon design of mobile terminal}

The design method of single-line icon. Single-line icon of mobile terminal refers that all the icon elements are reflected with pure lines, without adding any block element. First of all, it sketches out the draft of the icon with the pen in Photoshop or Illustrator according to the icon design process and then it is created by adding the strokes with corresponding width and setting the color. This single-line icon is suitable for the mobile phone desktop with unified tone, giving a light and bright visual experience to people. It can also be used in conjunction with monochrome shading, contributing to highlighting the details of the icon and the user experience.

The design method of silhouette icon. Silhouette icon of mobile terminal refers that all the body elements of the icon are manifested in the form of monochrome silhouette, which does not carry out multi-level design of the icon. The microphone of phone icon, the dialog box of information icon, the computed button of calculator icon, the notes of music icon, the letters of e-mail icon and the gamepad of game center icon are all designed and made in the form of monochrome silhouette. The design of silhouette icon of mobile terminal is completed by filling color of icon body elements based on the single-line icon of mobile terminal. It can not only be used together with circular contour, but also be used alone. It is also applicable to the mobile desktop with unified tone, which emphasizes the contrast of the icon and the background, and highlights the body elements of the icon.

The design method of imitation seal icon. Imitation seal icon of mobile terminal refers that all body elements of icons are reflected in the white or hollow form, which only fills the icon outline by monochrome. The icon is presented just like the colorful incised seal, highlighting the immersing design method. The overall icon has a strong tableau sense, which is easy to unify the form. Imitation seal icon of mobile terminal is produced by filling all the elements of the icon based on single-line icon of mobile terminal, which has no specific requirements on the phone desktop.

The design method of long shadow icon. Long shadow icon of mobile terminal refers to the icon which adds monochrome transparent shadow with 45 or 135 degree angle to the body elements of the icon, whose shadow length reaches the edge of the icon contour. The body elements such as microphone, dialog box, computed button, notes, letters, and gamepad in the icon all add to the effect of monochrome shadow. Long shadow icon of mobile terminal is created by adding shadows to the body elements of the icon based on the flat app icon, and the body elements of the icon can add to the shadow effect respectively. In Illustrator, we can create long shadows by using the path finder or the hybrid options. In Photoshop, we can create long shadows by using the pen or the shape layer. The long shadow icon highlights the body elements of the icon in a simple and clean way, increasing the sense of level cleverly.

The design method of projection icon. The projection icon of mobile terminal refers to the icon adding small displacement projection to the body elements of the icon. The projection elements do not add eclosion and gradient, emerging in the form of two-dimensional elements. The projection icon of mobile terminal is produced by adding projection to the body elements of the icon on the basis of flat app icon. The projection angle had better to be 45 degree angle, and the transparency should be controlled around $20 \%$ to $50 \%$. In Illustrator or Photoshop, we can create shadow by using the special effect of projection. Such icon should pay attention to the displacement parameter of shadow, and ensuring that the shadow is not out line with the body elements is the key to success.

\section{Summary}

With the constant increase of product type and user demand, it is necessary to have novel design 
language to meet the diversified demands of products and users. The accelerated pace of human life requires high accuracy of information transmission, and currently consumers' visual environment is full of various complicated designs, so obviously Skeuomorphism is no longer favored by people. For the time being, the flat design of simple interface and clear presentation is more consistent with people's taste. Adhering to the design principle of putting people first, it focus on the function of the product, with complete new interactive experience mode. At present, Skeuomorphic design is near saturation, and new Skeuomorphic design is going with the flow, which will be the mainstream style of interface design for some time.

\section{References}

[1] JiangDawei, The rise of UI design in modern style, Modern Decoration ，2012(09):182-184

[2] Wayne,Borody,ShaoMing,Japanese robot scientist Mori Masahiro's Buddhist concept of "terror Valley", Journal of Yibin University 2012(8): 1-7

[3]SongFang,JinJinghong,DaiXinhui, Analysis of the "flat" mobile phone interface design ,Packaging Engineering, 2012, 33(014): 60-63 